\title{
A novel intron mutation in $F B N-1$ gene identified in a pregnant woman with Marfan syndrome
}

\author{
Yuduo $\mathrm{Wu}^{1,2,3^{\dagger}}$, Hairui Sun ${ }^{2,3,4 \dagger}$, Yihua He $\mathrm{e}^{2,3,4^{*}}$ and Hongjia Zhang ${ }^{1,2,3^{*}}$
}

\begin{abstract}
Marfan syndrome (MFS) is one of the most common hereditary connective tissue diseases, with great individual heterogeneity. We reported a Chinese pregnancy with Clinical diagnosis of MFS, performed whole-exome sequencing, and screened for the genetic abnormality. We also conducted an in vitro mini-gene splicing assay to demonstrate the predicted harmful effects of an intronic variant of FBN-1. Exome sequencing identified a novel intronic variant (c.6497-13 T>A) in intron 53 of the FBN-1 gene (NM_000138.4). It's predicted to insert 11 bp of intron 53 into the mature mRNA. The mini-gene splicing experiment demonstrated that c.6497-13 T>A could result in 11 bp retention in intron 53 to exon 54 (c.6496_6497ins gtttcttgcag) and the use of an alternative donor causing the frameshift p.Asp2166Glyfs*23. According to the results, the pregnant woman chose to continue the pregnancy and gave birth to a healthy baby. This study expands the genetic mutation spectrum of MFS patients and indicates the importance of intron sequencing.
\end{abstract}

Keywords: Marfan syndrome, FBN-1 gene, Splicing mutation, Mini gene, Sequencing

\section{Introduction}

Marfan syndrome (MFS) is an autosomal dominant connective tissue disease with an incidence of 2-3/10000 without any ethnic, geographic, or professional orientation [1]. The condition caused by mutations in the human fibrillin-1 (FBN-1) gene [2]. More than $3000 \mathrm{FBN}-1$ site mutations have been reported. The mutation spectrum is composed of nonsense, missense, frameshift, and exon deletion, etc. [3] Among them, about 90\% of MFS patients' mutation sequence information limited to the coding region and specific splice sites. However, about $10 \%$ of patients still cannot identify the genetic cause of the disease [1]. Part of the reason may be that

\footnotetext{
*Correspondence: yihuaheecho@163.com; zhanghongjia722@ccmu.edu.cn †Yuduo Wu and Hairui Sun are joint first authors.

${ }^{2}$ Key Laboratory of Medical Engineering for Cardiovascular Disease, Ministry of Education, Beijing, China

'Department of Cardiac Surgery, Beijing Anzhen Hospital, Capital Medical University, No.2, Anzhen Road, Chaoyang District, Beijing 100029, China Full list of author information is available at the end of the article
}

advances in sequencing have not significantly affected the clinical interpretation of uncertain significance variants, such as intron variants that may affect splicing [4, 5]. This study, reports a pregnant woman with a clinical diagnosis of MFS, who carried a novel intron mutation (NM_000138.4:c.6497-13 T>A). Through the in vitro characterization of the effect of this mutation on mRNA, we determined that it was a pathogenic mutation and used it for the patient's family management, and successfully gave birth to a normal baby. The report is as follows.

\section{Case presentation \\ Patient information}

Proband female: 33 years old, height $183 \mathrm{~cm}$, weight 60 $\mathrm{kg}$, the patient admitted to the cardiac surgery department of our hospital because of a sudden aortic dissection, and the physical examination found: slender limbs, positive wrist signs, thoracic deformity, high myopia, as 

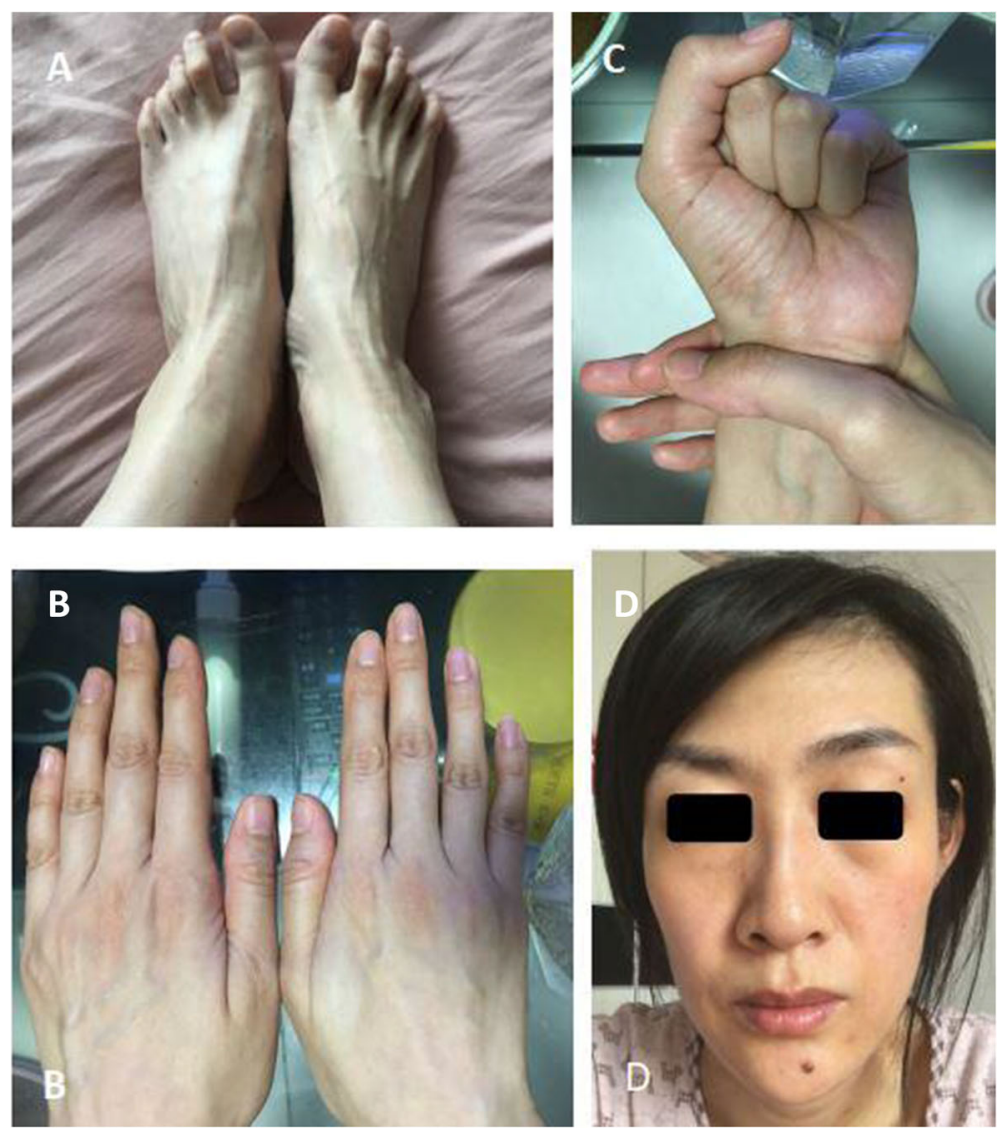

Fig. 1 Arachnodactyly of the proband (a and $\mathbf{b})$; positive wrist (c); characteristic face with dolichocephaly, malar hypoplasia, enophthalmos, myopia more than 3 diopters $(\mathbf{d})$

shown in Fig. 1. Ultrasound examination revealed: widening of the aortic root, mitral valve prolapse, clinical suspected MFS, surgical treatment, no genetic testing. Last year, the patient was pregnant and had another outpatient consultation. Family status: The family has three generations, five people, and one patient (the proband herself), as shown in Fig. 2. The proband's parents and younger brother have no clinical symptoms of MFS, and both parents have no relatives with relevant symptoms.

\section{Molecular findings}

A trio (proband and her parents) whole-exome sequencing was performed using methods described previously [6]. Whole-Exome sequencing identified a novel variant, c.6497$13 \mathrm{~T}>\mathrm{A}$, in intron 53 of the FBN-1 gene (NM_000138.4). Subsequent Sanger sequencing confirmed that the mutation was heterozygous in the pregnant woman (Fig. 3) and wildtype in her parents, younger brother, and fetus.

\section{In silico splicing analysis}

Computational predictions conducted using HSF (http:// www.umd.be/HSF3/HSF.html) revealed that the intronic c.6497-13 $\mathrm{T}>\mathrm{A}$ variant might influence the splicing

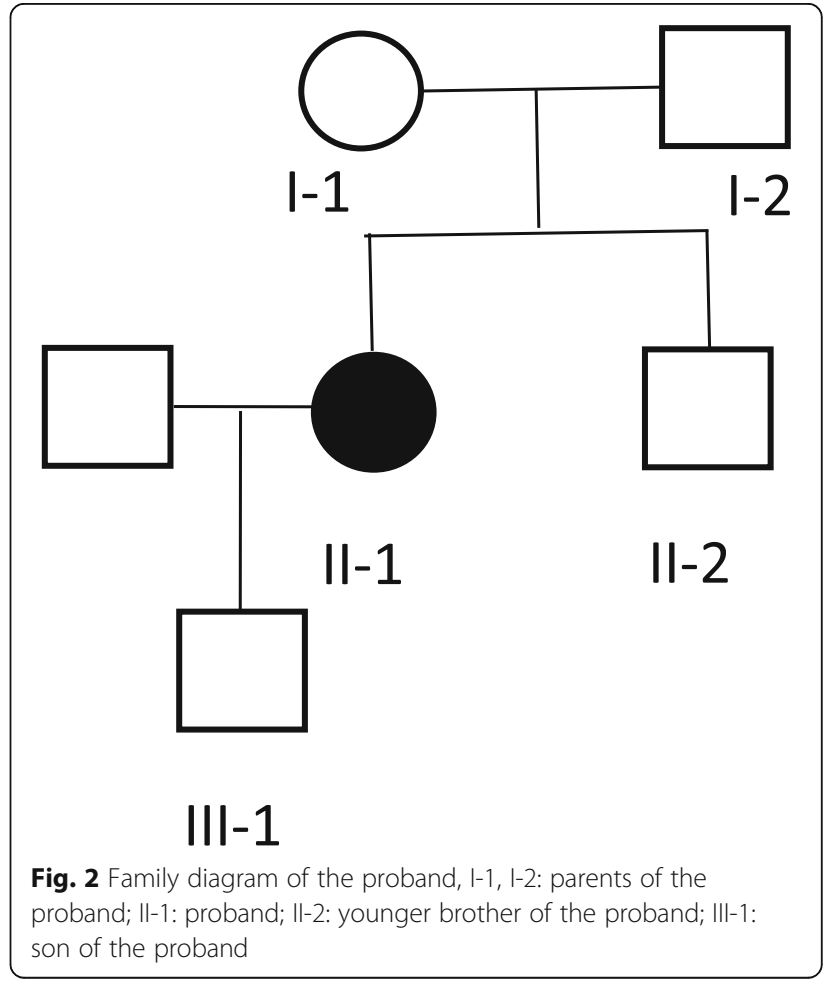




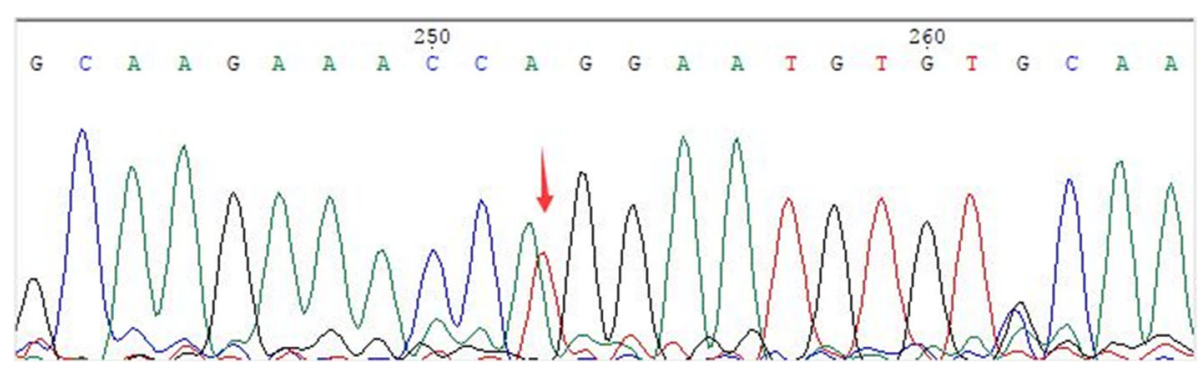

Fig. 3 Sanger sequence diagram of the proband, the red arrow points to the mutation site. Polymerase chain reaction (PCR) amplication was performed using forward primer (caactcctgtgagctgttgc) and reverse primer (acgttgtccacagtgagtcc). The obtained sequence was compared with the FBN-1 reference gene (NM_000138.4) to identify mutations

process by differentially affecting canonical versus cryptic splice site utilization. The newly detected variant predicted to insert $11 \mathrm{bp}$ of intron 53 into the mature mRNA (Fig. 4-a). This variant should give rise to a frameshift and a premature termination codon (p.Asp2166Glyfs*23).

\section{Results of the mini-gene splicing assay}

To prove that the c.6497-13 T>A variant caused the 11 bp insertion, we then conducted the mini-gene splicing experiment using RNA from blood lymphocytes as described previously [7]. The mini-gene splicing products were analyzed by PCR amplification with plasmidspecific primers and visualized with polyacrylamide gel electrophoresis. The electrophoresis results of wild-type and c.6497-13 $\mathrm{T}>\mathrm{A}$ transfections indicated that the mutant band was bigger than the wild-type, and the migration became slower (Fig. 4-b). The amplicons confirmed by Sanger sequencing showed that the cDNA fragment obtained from the c.6497-13 T>A plasmid contained an additional $11 \mathrm{bp}$ in intron 53 to exon 54 (c.6496_6497ins gtttcttgcag) (Fig. 4-c). This result is consistent with the in silico analysis result.

\section{Screening of other genes associated with thoracic aortic aneurysm and dissection}

Besides finding the $F B N-1$ mutation presented above, we also analyzed variants in genes associated with thoracic aortic aneurysm and dissection (Supplementary Table 1). No pathogenic/likely pathogenic variants were identified in these genes.

\section{Discussion}

This study describes a pregnancy with Clinically diagnosed MFS that carried a novel intronic variant in the $F B N-1$ gene. We added this novel variant to the $F B N-1$ mutational repertoire and demonstrated this variant's effect at the mRNA level by in vitro Mini-gene splicing assay. Furthermore, Based on the results of genetic tests, pregnancy decisions are guided.

This novel intronic variant in the FBN-1 gene (c.6497$13 \mathrm{~T}>\mathrm{A}$ ) has previously not been reported as pathogenic or benign and has not observed in the general population (gnomAD: https://gnomad.broadinstitute.org). It showed a deleterious effect by multiple in silico algorithms. Furthermore, in vitro experiment of mini-gene splicing assay verified that the variant alters splicing, leading to a frameshift and a premature termination

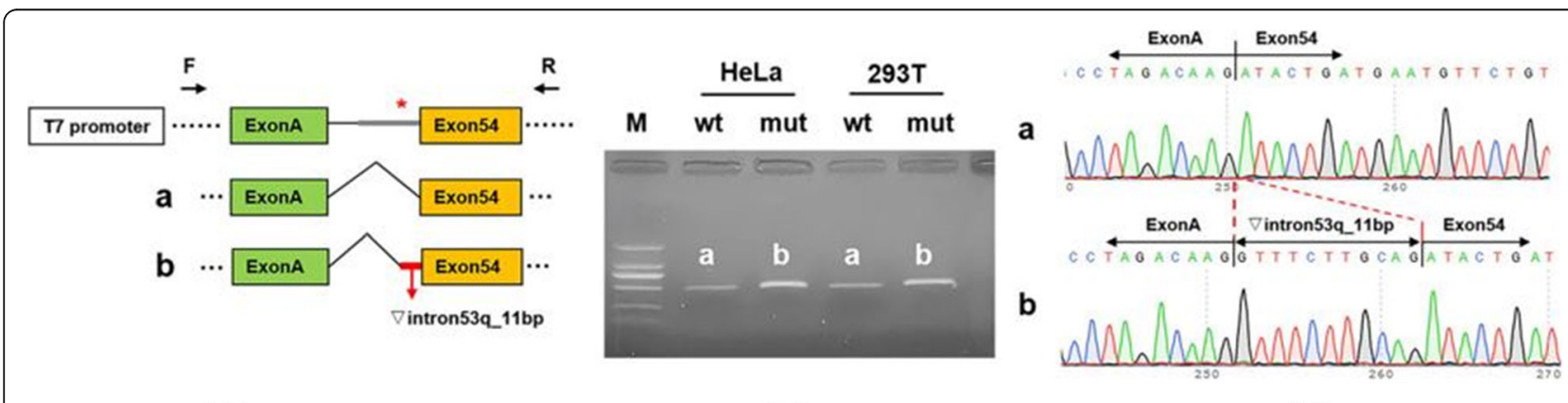

(A)

(B)

(C)

Fig. 4 Results of the mini-gene splicing assay. a Schematic diagram of mini-gene construction and the c.6497-13 T>A variant related abnormal splicing. The asterisk indicates the location of the c.6497-13 T>A variant. b Gel electrophoresis of RT-PCR products: the band of the mutant was bigger than the wild-type. c Mini-gene product sequencing results: a The wild-type mini-gene (FBN1-wt) formed normal mRNA composed of exon54; b The mutant mini-gene (FBN1-mut) caused a splicing abnormality, resulting in the retention of the 11 bp in intron 53 
codon. It is a loss-of-function variant and expected to result in either an abnormal truncated protein product or loss of protein from this allele through nonsensemediated mRNA decay. Following the revised Ghent nosology for the MFS, the c.6497-13 T>A variant was pathogenic. In this work, we highlighted the importance of including extended intronic regions in analysizing WES (whole-exome sequencing, WES) results during variant prioritization and post-analytical phase for clinical purposes. This work also points out the need to introduce functional investigations to verify further the pathogenicity of intronic variants with a potential pathogenic effect.

Through this study, we also found that the diagnosis of MFS through genetic testing has limitations. Despite advances in genetic testing technology, about $10 \%$ of patients still cannot be diagnosed through genetics. According to our case and some previous scattered case reports, it's suggeste that part of the reason is the variation of introns or other non-coding regions [8]. On the other hand, it is necessary to consider the differentiation from other hereditary macrovascular diseases, such as: Loeys-Dietz Syndrom, Ehlers-Danlos Syndrom, etc. [9] We found no relevant mutations in the case.

For MFS patients with atypical clinical phenotypes, genetic testing can not only diagnose the disease but also distinguish it from other genetic macrovascular diseases with similar phenotypes. Through large-scale testing within the family, it is also possible to find patients with gene mutations without clinical phenotypes and help them prevent adverse aortic events in advance. At the same time, it can also provide fertility guidance for women of childbearing age. Like the pregnant women in this study, she performed amniocentesis in the second trimester. The genetic test results showed negative, and the pregnancy continued, giving birth to a healthy baby.

Because both parents are not the carrier for the mutation, the proband's mutation should be a de novo mutation in the family. Although the proband gave birth to a healthy baby, if this patient becomes pregnant again in the future, the probability of MFS in the second child is still $50 \%$ [1]. Therefore, we recommended that this part of patients undergo amniotic fluid puncture during the second trimester of pregnancy and check the karyotype.

In conclusion, this study enlarges the mutation spectrum of $F B N-1$ and points out the importance of intronic sequence analysis and the need for integrated functional studies in $F B N-1$ diagnosis. The literature on $F B N-1$ intronic variants affecting non-typical splice sites is currently limited to single reports [10]. Therefore, the large sample size and large cohort study need to carry out. To further reveal the significance of non-coding region (intron) variation to MFS.

\section{Supplementary Information}

The online version contains supplementary material available at https://doi. org/10.1186/s41065-020-00170-w.

Additional file 1: Supplementary Table 1. Genes associated with thoracic aortic aneurysm and dissection.

\section{Abbreviations}

MFS: Marfan syndrome; WES: whole exome sequencing; PCR: Polymerase chain reaction

\section{Acknowledgments}

This study complied with the Declaration of Helsinki and approved by the Ethics Committee of Anzhen Hospital, Capital Medical University. The proband and all family members signed an informed consent form. The personal information of the proband and all family members is strictly confidential. We thank our laboratory for helping for the biochemical evaluation of the patient and the families for their kind availability in sharing the scientific community's findings.

\section{Authors' contributions}

Yuduo Wu was mainly responsible for the article's writing and case collection, Hairui Sun was mainly responsible for the gene sequencing part of the article; Yihua He and Hongjia Zhang were mainly responsible for the overall thinking and fund support of the article. The author(s) read and approved the final manuscript.

\section{Funding}

This work was supported by the National Key R\&D Program of China (2018YFC1002300) and Beijing Municipal Administration of Hospitals'Ascent Plan (DFL20180602)

Availability of data and materials

Availably.

\section{Competing interests}

All authors declare no conflict of interest concerning this work.

\section{Author details}

${ }^{1}$ Department of Cardiac Surgery, Beijing Anzhen Hospital, Capital Medical University, No.2, Anzhen Road, Chaoyang District, Beijing 100029, China. ${ }^{2}$ Key Laboratory of Medical Engineering for Cardiovascular Disease, Ministry of Education, Beijing, China. ${ }^{3}$ Beijing Key Laboratory of Maternal-Fetal Medicine and Fetal Heart Disease, Beijing Anzhen Hospital, Capital Medical University, Beijing, China. ${ }^{4}$ Ultrasound Department of Beijing Anzhen Hospital, Capital Medical University, No.2, Anzhen Road, Chaoyang District, Beijing 100029, China.

Received: 6 September 2020 Accepted: 15 December 2020

Published online: 06 January 2021

\section{References}

1. Verstraeten A, Alaerts M, Van Laer L, Loeys B. Marfan syndrome and related disorders: 25 years of gene discovery. Hum Mutat. 2016;37:524-31.

2. Lee B, Godfrey M, Vitale E, Hori H, Mattei M, Sarfarazi M, Tsipouras P, Ramirez F, Hollister D. Linkage of Marfan syndrome and a phenotypically related disorder to two different fibrillin genes. Nature. 1991;352:330-4.

3. Xu S, Li L, Fu Y, Wang $X$, Sun H, Wang J, Han L, Wu Z, Liu Y, Zhu J, Sun L, Lan F, He $Y$, Zhang H. Increased frequency of FBN1 frameshift and nonsense mutations in Marfan syndrome patients with aortic dissection. Mol Genet Genomic Med. 2019;8:e1041.

4. Torrado M, Maneiro E, Trujillo-Quintero J, Evangelista A, Mikhailov A, Monserrat LJB. A Novel Heterozygous Intronic Mutation in the Gene Contributes to RNA Missplicing Events in the Marfan Syndrome. Biomed Res Int. 2018;2018:3536495

5. Kayhan G, Ergun MA, Ergun SG, Kula S, Percin FE. Identification of three novel FBN1 mutations and their phenotypic relationship of Marfan syndrome. Genet Test Mol Biomarkers. 2018;22:474-80.

6. Sun H, Yi T, Hao X, Yan H, Wang J, Li Q, Gu X, Zhou X, Wang S, Wang X Wan P, Han L, Chen J, Zhu H, Zhang H, He Y. Contribution of single-gene 
defects to congenital cardiac left-sided lesions in the prenatal setting Ultrasound Obstet Gynecol. 2020;56:225-32.

7. Yanyun Wang YS, Liu M, Zhang X, Jiang T. Functional Characterization of Argininosuccinate Lyase Gene Variants by Mini-Gene Splicing Assay. Front Genet. 2019;10:436.

8. Fusco C, Morlino S, Micale L, Ferraris A, Grammatico P, Castori M.

Characterization of two novel Intronic variants affecting splicing in FBN1related disorders. Genes. 2019;10:442.

9. Cheulot P, Saucedo M, Bouvier-Colle MH, Deneux Tharaux C, Kayem G Mortalité maternelle chez les patientes atteintes de syndromes de Marfan ou d'Ehlers-Danlos vasculaire en France, 2001-2012. Gynécol Obstét Fertil Sénol. 2019;47:30-5.

10. Wypasek E, Potaczek D, Hydzik M, Stapor R, Raczkowska-Muraszko M, Weiss J, Maugeri A, Undas AJC. Detection and a functional characterization of the novel FBN1 intronic mutation underlying Marfan syndrome: case presentation. Clin Chem Lab Med. 2018;56:87-91.

\section{Publisher's Note}

Springer Nature remains neutral with regard to jurisdictional claims in published maps and institutional affiliations.

Ready to submit your research? Choose BMC and benefit from:

- fast, convenient online submission

- thorough peer review by experienced researchers in your field

- rapid publication on acceptance

- support for research data, including large and complex data types

- gold Open Access which fosters wider collaboration and increased citations

- maximum visibility for your research: over $100 \mathrm{M}$ website views per year

At BMC, research is always in progress.

Learn more biomedcentral.com/submissions 